\title{
Marianne Leroy (1947-2008)
}

\author{
Marie BONNAFÉ*
}

*Psychiatre adjoint des Hôpitaux (honoraire), Psychanalyste, membre titulaire (hon.) de la SPP, Présidente d'ACCES', Paris, France. marie.bonnafe@ yahoo.fr

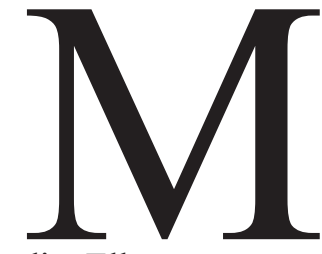

arianne Leroy nous a quitté, bien trop tôt, il y a une année, en janvier 2008 à soixante-et-un ans, après avoir avec courage surmonté une longue maladie. Elle reste pour nous, comme le dit le poète qu'elle aimait, parmi « Ceux qui nous aident à vivre » (Paul Eluard) et tous ceux qui l'ont connue disent d'abord sa capacité d'altérité, sa vive sensibilité et son talent à transmettre sa culture si étendue.

Lorsque je pense à elle c'est un autre texte qui me vient « L'art de la sympathie »[1]. Elle a représenté je crois un modèle exemplaire dans sa génération de personnalités aussi discrètes qu'obstinées dans leur projet humain et professionnel, qui ont œuvre à renverser l'image des êtres affectés de troubles psychiques qui, de fous, malades mentaux, sont devenus des personnes à part entière, nos patients.

Suivre sa démarche et son engagement professionnel, lire ses articles devra être je crois une bonne école pour nous tous, pour pouvoir poursuivre ces enjeux toujours menacés, toujours à recommencer.

Sa vocation en psychiatrie est née dès son enfance, auprès de Lucien Bonnafé, inspirateur et ami de tout temps (elle écrira l'interview de lui dans la revue Synapse [2]). Sur les ruines de l'Hôpital Psychiatrique de Sotteville-les-Rouen bombardé il va, avec de bien petites équipes, construire les bases de la «psychiatrie hors les murs » en Seine Maritime ou il s'engage activement. C'est là que toute la fine fleur de la nouvelle génération psychiatrique de l'après-guerre vient refaire le monde. On y parle Entretiens de Bonneval [3], psychanalyse, engagement des syndicats, nouvelles architectures, culture poésie Leçons que retiendra la jeune psychiatre venue en région parisienne. Marianne Leroy se définit par un attachement à ces changements dans le service public, avec une totale priorité $\mathrm{du}$ lien individuel avec chaque patient, comme le montreront ses publications qui privilégient toujours les cas cliniques pris dans des situations institutionnelles. Ainsi que le dit Jean Charles Pascal qui évoque sa sensibilité maîtrisée très remarquable avec les patients et «sa capacité à repérer les variations les plus ténues dans les affects qui lui a permis de théoriser sur « l'infra »-clinique dans son article sur « Mélancolie et clinique de jour » où elle interprète les « petits riens du quotidien $\gg$.

Son parcours est significatif de ses choix : Interne à la Fondation Vallée dans l'équipe du Professeur Misès puis au CHS de la Queue en Brie chez le Dr Couvreur et $\mathrm{M}^{\mathrm{me}}$ le Dr Sarradin, Médecin consultant à l'UDSM de Fontenaysous-Bois, ensuite au CMPP d'Aubervilliers. Jeune médecin, elle fait des remplacements en médecine générale, expérience qu'elle évoquait souvent pour la relier à son travail clinique. Puis elle commence une longue collaboration avec le Dr J.C. Pascal à la faveur du passage de celuici comme Assistant dans le service de Lucien Bonnafé qui la lui fait connaître. D'abord Interne dans son Service à Clermont d'Oise, qui émerge de ses structures archaïques, elle le suit dans le service du IX ${ }^{\mathrm{e}}$ Secteur des Hauts de Seine, d'abord Interne à l'Hôpital Erasme, elle devient en 1976 le médecin responsable de l'Hôpital de jour Jean Wier à Puteaux. « Dans cette structure particulièrement complexe qu'est un hôpital de jour d'un secteur de psychiatrie générale, elle développe Jean Wier dans un esprit de recherche et d'adaptation d'offre de soin, sans sectarisme mais dans le respect de ses convictions, écrit J.C. Pascal, et c'est bien auprès de tous que Marianne sait utiliser son rayonnement personnel au bénéfice des patients qui présentent les pathologies les plus lourdes ». " Elle ouvrait

1. ACCES, Actions Culturelles Contre les Exclusions et les Ségrégations, www.acces-lirabebe.fr 
très tôt un débat sur ce qui reste très actuel sur l'utilisation des prises en charge à temps partiel, avec la capacité d'associer une réflexion psychopathologique subtile aux modalités du soin en Institution ». Elle crée avec l'équipe une structure adaptée à une approche actuelle, psychiatrique et psychanalytique, comme tous ceux et celles avec qui elle collabore en témoignent avec chaleur - et comme elle l'a écrit dans son bel article [4]. Tout en restant très au fait des prescriptions médicamenteuses, elle ne les concevaient pas sans l'accompagnement de la psychothérapie psychanalytique permettant que le patient retrouve son équilibre propre dans ses nouvelles restructurations psychiques

À côté de ses responsabilités à Jean Wier, elle prend en 1983 la direction du CMPP de la Grange Batelière - également [5] BAPU pour les étudiants. L'Association de réadaptation gère aussi un hôpital de jour pour adolescents : il s'agit donc d'un dispositif faisant fonction de public - en liaison avec les activités du secteur psychiatrique qu'elle consolide. Ici encore, Marianne laisse sa patte, améliorant le fonctionnement démocratique de l'équipe : en répartissant le premier entretien entre tous les médecins - et non au seul médecin directeur - étendant plus largement à l'ensemble des praticiens la pratique des psychothérapies. Son aptitude à diriger dans un grand respect de chacun est par tous appréciée. Le Dr Jean Pierre Pinel se souvient que « l'on pouvait lui confier des cas difficiles, voire vraiment atypiques, qui ne décourageait jamais son inspiration ».

Avec la lourde charge de cette direction, elle quitte à regret Jean Wier, et elle va travailler à temps partiel à l'EMP de Gagny consacrant son attention aux enfants mais aussi aux familles et à l'environnement, suivant encore l'enseignement du « désaliénisme » : le problème de l'enfant 《 arriéré »-devenu « handicapé »-, c'est avant tout l'entourage.

Tous se souviennent de sa passion pour la clinique alliée à sa passion de la culture. Suivant les séminaires de F. Dolto, G. Haag, Rosenfeld bien d'autres, sans sectarisme en effet. Elle a entrepris une analyse personnelle, a toujours fait ensuite des thérapies analytiques, restant indépendante, non engagée dans une École psychanalytique, mais appréciée de tous dans sa pratique, elle témoigne d'un engagement psychanalytique vivant, dans la chair même, pourrait-on dire, de la relation humaine. Surtout elle échangeait constamment avec un réseau de collègues engagés dans un même esprit, un même regard sur les patients. Je me souviens d'un été, en vacances dans le midi Marianne apprend qu'une jeune artiste (dont elle connaît la famille), en tournée à l'étranger, y est hospitalisée pour une bouffée délirante. En un délai record, elle trouve un point de chute pour son retour, établi un contact approfondi avec les collègues de là-bas et d'ici, soutient la patiente et la famille et tout cela sans que rien ne paraisse lui peser dans notre groupe de vacances familiales. Combien de fois l'ai-je vue établir ainsi de tels contacts et combien de fois l'ai-je utilisée !

Tous rappellent ses ouvertures si étendues à la culture, marquant toute sa vie, liés à ses engagements - ce qui l'a sans nul doute soutenue dans son long combat contre la maladie, qu'elle a si longtemps surmonté et où elle n'a jamais abdiqué. Indissolublement liée à sa chaleur humaine, avec son empathie, ses amitiés, ses liens familiaux et avant tout pour ses deux fils, Jean Philippe et Hadrien. En premier son intérêt pour théâtre, auquel elle fut liée dés sa jeunesse, avec Jean Pierre Jorris, qui avait fait ses début au TNP à Chaillot. Ses intérêts si étendus mais surtout ce talent personnel à être passeuse de culture, que ce soit la chanson ou la peinture, mais plus encore sa priorité d'atteindre ce qui en chacun de nous nous touche le mieux et nous lie à la communauté. Si elle respectait profondément l'héritage de nos pères elle a très fermement construit sa vie indépendante. Elle n'adhérait pas à un parti, - même si elle aimait avoir ses entrée libres au journal L'Humanité, dont son père, Roland Leroy était le directeur. Il me semble qu'elle avait recueilli toute l'ouverture à la culture d'une tradition combative ouvrière sans en subir de servitudes. Son engagement dans la voie de la psychiatrie psychanalytique dans la filiation de Lucien Bonnafé revêtait pour elle un engagement profond. Certes avec l'élaboration de changements sociaux, mais surtout, pour ce qui était de ses orientations personnelles, avec des remaniements profonds dans la répartitions des biens culturels et cela avec la profonde tolérance qui la caractérisait. Je termine en la laissant nous parler elle même, avec son texte lors du cinquantenaire du CMPP de « La Grange ».

« J'avais amené cette mère à comprendre que l'espace de consultation pouvait devenir pour elle un espace de pensée, d'élaboration, d'aide et non d'assistance, et elle pu alors confier ses difficultés et parler de sa propre histoire » et, plus loin : «Communiquer autour d'un poème avec un parent peut paraître trop anodin - alors que la prise de contact avec ce père a été plus que difficile -, mais je pense que cela a été déterminant pour la suite, dans cette pratique de la consultation thérapeutique. $)$

Il est des noms qui nous font vivre... 


\section{RÉFÉRENCES BIBLIOGRAPHIQUES}

1. Leroy M. Mélancolie et clinique de jour. Journées de Sèvres. L'information Psychiatrique $1987 ; 63$ ( $n^{\circ}$ spécial mars).

2. Leroy M. Entretien avec Lucien Bonnafé. Synapse janvier 1990.

3. Leroy M. Le temps partiel en hôpital de jour, mode d'abord de la prise en charge des schizophrènes. Journées de Saint Malo. L'information Psychiatrique octobre 1994.
4. Leroy M. Les effets thérapeutiques de la consultation. Centre Claude Bernard, le Cinquantenaire du CMPP.

5. Les entretiens de Bonneval (sous la direction de Henri Ey). L. Bonnafé, H. Ey, S. Follin, J. Lacan J. Rovard. Le problème de la psychogenèse des névroses et des psychoses. Paris : Editions Desclée de Bouvier, 1950.

6. Bonnafé L. Le personnage du psychiatre. L'art de la sympathie. Conférence de L'Evolution Psychiatrique le 25 mars 1947. Évolution Psychiatrique, 18 textes de L. Bonnafé, n 1/1969.

\section{Communiqué \\ COLLOQUE \\ GYPSY IX \\ 2009}

"Recherche bonheur désespérément... "

\section{4 et 5 décembre 2009 \\ Faculté de Médecine \\ Amphithéâtre Binet \\ 45 rue des Saints Pères \\ 75006 Paris, France}

La réflexion sur la pensée du bonheur n'a jamais cessé. Pourtant, aujourd'hui, si l'idée et l'aspiration à une vie meilleure transcendent le temps et l'espace, ces données ne suffisent plus à nourrir la notion de bonheur. Sommes-nous devenus nos propres tyrans, assiégés par l'idéologie environnante, recherchant l'efficacité et contraints à la recherche du plaisir, de la performance. Tout est organisé, essais, discours, films... pour être écartelés entre rêve de bonheur et brutalité de l'existence. Illusion et désenchantement. Si la réalisation des désirs est contrariée par l'échec devant la réussite il n'y a pas d'apaisement et la douleur psychique reste intense. Le bonheur est devenu un impératif collectif, un devoir, une obligation telle que sonabsence serait ressentie comme une blessure béante. N'estelle pas devenue culpabilisante, cette idée du bonheur, si on ne la réalise pas? L'idée de bonheur serait-elle tributaire d'un sentiment de culpabilité inconsciente? Alors, celui qui s'adresse au psychanalyste, lui demande til le bonheur? Comme on demanderait la lune ? Le psychanalyste pourrait-il lui promettre, sans se vivre comme un charlatan, qu'une fois débarrassé de ses symptômes, il serait en mesure d'apprécier le monde, la vie comme un paradis?

\section{Organisation \\ Pr René Frydman \\ Dr Muriel Flis-Trèves}

\section{Orateurs}

Dr ANDRE Christophe, Psychiatre Mme AGACINSKI Sylviane, Philosophe Mr ASSOUN Paul-Laurent, Psychanalyste Mme BLOCH-DANO Evelyne, Écrivain Mme DARRIEUSSECQ Marie, Écrivain Mme DESARTHE Agnès, Écrivain

Dr DOLTO Catherine, Haptothérapeute Dr HEFEZ Serge, Psychiatre-Psychanalyste Mr HIRSCH Martin, Haut commissaire au Gouvernement

Mr ENTHOVEN Raphaël, Philosophe

Dr FLIS-TREVES Muriel , Psychiatre-Psychanalyste

Pr FRYDMAN René, Gynécologue-Obstétricien

$\operatorname{Pr}$ MIMOUN Maurice, Chirurgie réparatrice

Dr ROSENBLUM Ouriel, Psychiatre-Psychanalyste

Dr POMMEREAU Xavier, Psychiatre

Dr RAYNA Claude, Psychiatre-Psychanalyste

Mme SCHNECK Colombe, Journaliste - Écrivain

Dr TASSIN Jean-Pol, Neuropharmacologue

Mme THOMPSON Caroline, Psychanalyste

Renseignements

JS Congrès, 39-41, rue Louis Blanc, 92038 Paris La Défense, France.

Tél : 0143347671 -Fax : 0147176860 -E-mail : jscongres@wanadoo.fr 